\title{
Accuracy of Swanepoel Method in Calculation of Polymer Film Thicknesses
}

\author{
SARUnRit Kesornkhup ${ }^{a}$, Adisorn Tuantranont $^{b}$, TAnOm Lomas $^{b}$, \\ Chakrit SRIPrachuabWOng ${ }^{b}$ And KAmOL WASAPINYOKUL ${ }^{a, c, *}$
}

${ }^{a}$ College of Advanced Manufacturing Innovation, King Mongkut's Institute of Technology Ladkrabang, 1, Chalongkrung 1 Road, Ladkrabang, Bangkok 10520, Thailand

${ }^{b}$ National Electronics and Computer Technology Center (NECTEC), 112, Phahonyothin Road, Khlong Luang, Pathumthani 12120, Thailand

${ }^{c}$ Faculty of Information Technology, King Mongkut's Institute of Technology Ladkrabang, 1, Chalongkrung 1 Road, Ladkrabang, Bangkok 10520, Thailand

Received: 28.05.2021 \& Accepted: 26.07.2021

Doi: $10.12693 /$ APhysPolA.140.113

*e-mail: kamol.wa@kmitl.ac.th

\begin{abstract}
We studied the accuracy of the Swanepoel method in the calculation of thicknesses of spin-coated poly(methyl methacrylate) films with thicknesses up to $2500 \mathrm{~nm}$. Their thicknesses were calculated by using the Swanepoel method and subsequently compared with the measured actual values. Results showed that both thicknesses followed identical trends where films with higher solution concentrations or slower spin-coating speeds were thicker. The relative difference between the Swanepoel and the actual thicknesses was explained through the thickness of the flat region where the interference of transmitted light occurred. For a film whose flat region was thinner or thicker than other features of the film, its Swanepoel thickness was lower or higher, respectively, than the actual average value. Errors of the Swanepoel thicknesses from the actual values were analysed to find their correlation with the film surface roughness and thickness. When the film roughness and thickness increased fivefold, the error increased threefold and 1.6 times, respectively, indicating that the effect of the film roughness was predominant. Mathematically, this effect was the result of the fact that when roughness increased, the interference pattern shrank, and hence the values deviated. For the effect of thickness, thicker films had higher roughness, and consequently higher errors. Errors of as low as $5 \%$ and $0.86 \%$ were observed for films with the roughness of less than $15 \mathrm{~nm}$ and those with the thickness of $1800 \mathrm{~nm}$, respectively. This showed that the method can be used to calculate the thickness of $\mu$ m-thick polymer films, with a good level of roughness, with satisfying accuracy.
\end{abstract}

topics: Swanepoel method, film thickness, polymer film, spin-coating

\section{Introduction}

The thickness of a polymer film is a significant factor which affects the film's properties. In the case of films used for electrical purposes, these properties include the dielectric constant $[1,2]$ and resistivity $[3,4]$. Accordingly, there have been a number of reports on how film thickness could be varied to improve the performances of polymer-based thinfilm electronic devices including thin film transistors $[5,6]$ and capacitors $[7,8]$.

Film thickness is therefore a key parameter to be known in order to fabricate polymer thin-film devices and improve their performances. The parameter can be measured directly by several means including the use of a scanning electron microscope, or SEM, or a stylus profilometer. However, the sample-destructive natures of the said methods and the relatively high prices of the corresponding equipment restrict their uses. Apart from the direct measurement, several attempts have been proposed to calculate the film thickness by using other film's properties. These includes the use of experimental recipes, such as spin coating $[9,10]$ or sputtering $[11,12]$, to calculate the thickness of films deposited by each method.

Another non-destructive approach to calculate the film thickness is through the film's optical properties, the so called Swanepoel method. Firstly presented by Swanepoel [13] in 1983, the method was used to calculate the thickness of amorphous silicon layers. It has been used to calculate the film thickness by using the optical transmittance of the film. When light is incident onto the film, due to the multiple reflections inside the film, rays with different path lengths are transmitted through the film and interfere with one another. For a specific wavelength, the interference may be constructive or 
destructive - depending on the relations between such wavelength and the film thickness. This results in a wave-like pattern of the transmittance of a film over a range of wavelengths. With other properties, such as the refractive index of the substrate, the film thickness can be calculated.

The Swanepoel method has been used extensively to calculate the thickness of films made of several groups of materials, including metal oxides such as $\mathrm{Fe}_{3} \mathrm{O}_{4}[14]$ and $\mathrm{TiO}_{2}[15,16]$, and inorganic semiconductors such as $\mathrm{Ge}_{19} \mathrm{As}_{21} \mathrm{~S}_{60}$ [17], $\mathrm{Ge}_{25} \mathrm{Cd}_{5} \mathrm{Se}_{70}$ [18], and $\mathrm{As}_{40} \mathrm{~S}_{45} \mathrm{Se}_{15}$ [19]. However, the theory has some limitations in this. Firstly, the method is applicable only to a film with a good level of uniformity [20]. Even though there have been some methods proposed in a format of complicated mathematical models to include the surface roughness into the calculation [20], the trend of how the surface roughness affects the validity of the conventional Swanepoel model has not been widely studied empirically. Secondly, the method has mostly been studied on thin films with the thickness of less than $1 \mu \mathrm{m}[18,19,21,22]$. Only a few studies have been carried out on thick films, most of which are non-polymer materials [23]. There is still a lack of such a use of the method that allows direct calculation of the thickness of polymer films, hence striving to improve the method is still desirable.

In this paper, the thicknesses of polymer films fabricated by the spin-coating method with different spin-coating recipes were calculated by using the Swanepoel method. The modelled values, considered in the range of $400 \mathrm{~nm}$ to $2500 \mathrm{~nm}$, were subsequently compared with the actual values. Errors of the modelled values were calculated and analysed, especially for two factors - the film roughness and thickness - to figure out how each parameter affected the accuracy. The causes of errors were also discussed.

\section{Derivation of Swanepoel model}

Let us consider a transparent substrate with a smooth surface, uniform thickness, and a refractive index $s$, as shown in Fig. 1a. The substrate is coated with a film of a uniform thickness $d$, which is several orders of magnitude lower than the substrate's thickness. Light is incident onto the film at a smooth region. The incident light eventually transmits through the film and the substrate. Due to multiple reflections inside the film, as shown in Fig. 1b, the transmitted light would be the result of the multiple transmitted rays interfering with one another. The wave-like transmittance $T$ is a complex function of the refractive index of the film $n$, the refractive index of the substrate $s$ and the absorbance $x$ of the film, as follows [13]:

$$
T=\frac{A x}{B-C x \cos (\varphi)+D x^{2}},
$$
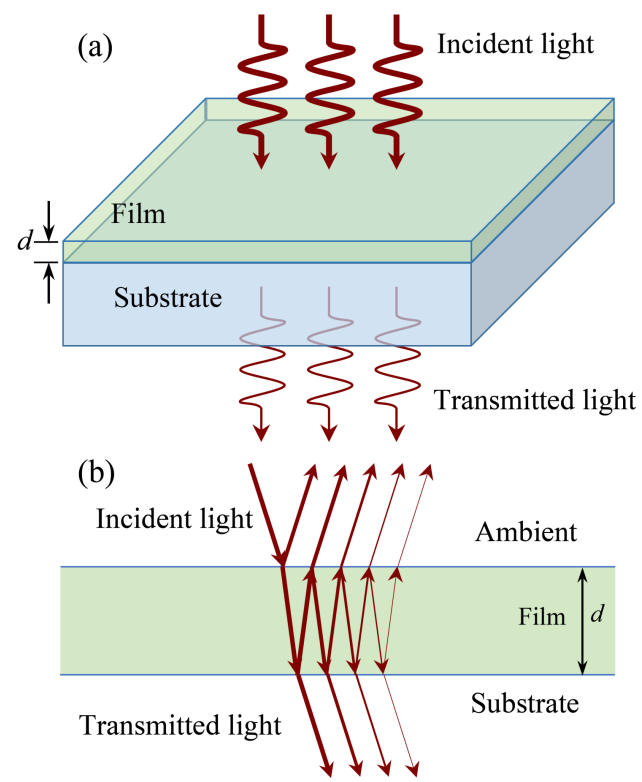

Fig. 1. Schematic diagrams of (a) a thin film on a thick substrate with light incident on and (b) multiple reflections of light in the film.

where $A, B, C, D$, and $\varphi$ describe the optical properties of the film and the substrate. The quantities are expressed as

$$
\begin{aligned}
& A=16 n^{2} s, \\
& B=(n-1)^{3}\left(n+s^{2}\right), \\
& C=2\left(n^{2}-1\right)\left(n^{2}-s^{2}\right), \\
& D=(n-1)^{3}\left(n-s^{2}\right), \\
& \varphi=\frac{4 \pi n d}{\lambda} .
\end{aligned}
$$

The film absorbance $x$ can be calculated from the absorption coefficient $\alpha$ of the film by using the following Lambert-Beer-Bouguer law [24], namely:

$$
x=\mathrm{e}^{-\alpha d} \text {. }
$$

Due to the wave-like shape of the transmittance wave $T$, it is covered by two envelope functions: the upper, $T_{M}$, and the lower, $T_{m}$, as shown in Fig. 2. For a highly transparent film, the transmittance spectrum can be separated, roughly, into two regions: the transparent and the weak absorption regions. In the former, the transmittance is high, i.e., the upper envelope function $T_{M}$ is nearly equal to one. In the latter, the transmittance is lower, i.e., the upper envelope function $T_{M}$ is slightly lower than one. This results in a wave-like pattern of constructive and destructive interferences aligned alternatively along the transmission spectrum, as shown in Fig. 2.

Using (1), $T_{M}$ and $T_{m}$ can be calculated as the functions with maximum and minimum values, respectively, as follows [13]:

$$
T_{M}=\frac{A x}{B-C x+D x^{2}},
$$


and

$$
T_{m}=\frac{A x}{B+C x+D x^{2}} .
$$

Both functions can be further derived for the transparent and weak absorption regions separately. For the transparent region, there is no observable absorption, and so $\alpha$ is assumably equal to zero, leading to $x$ equal to one in (3). Hence, the upper (4) and the lower (5) envelope functions are simplified, respectively, as:

$$
T_{M}=\frac{2 s}{s^{2}+1},
$$

and

$$
T_{m}=\frac{4 n^{2} s}{n^{4}+n^{2}\left(s^{2}+1\right)+s^{2}} .
$$

One can then rewrite (7) in the following way:

$$
n=\sqrt{M+\sqrt{M^{2}-s^{2}}},
$$

where $M$ is the property depending on $T_{m}$ and $s$, i.e,

$$
M=\frac{2 s}{T_{m}}-\frac{s^{2}+1}{2} .
$$

Both (8) and (9) clearly show that once $T_{m}$ and $s$ are known, $n$ at the wavelength $T_{m}$ can be calculated.

For the weak absorption, (4) and (5) can be simultaneously solved and rearranged so that they become independent of $x$ [13], i.e.,

$$
\frac{1}{T_{m}}-\frac{1}{T_{M}}=\frac{2 C}{A}
$$

Substituting $C$ and $A$ from (2) into (10), one yields

$$
n=\sqrt{N+\sqrt{N^{2}-s^{2}}},
$$

where $N$ is the property depending on $T_{M}, T_{m}$ and $s$, that can be expressed as follows:

$$
N=2 s \frac{T_{M}-T_{m}}{T_{M} T_{m}}+\frac{s^{2}+1}{2} .
$$

Note that for a set of peaks of the transmittance wave, where $T_{M}, T_{m}$ and $s$ are known, one can determine $N$ and $n$.

The calculation of the film thickness $d$ can be initiated from the basic equation for the interference fringes from normal incident light

$$
\begin{aligned}
& 2 n d=m \lambda \text {, } \\
& \text { or } \\
& d=\frac{m \lambda}{2 n},
\end{aligned}
$$

where $m$ is the integer stating the order of the concerned maximum.

Now, for two adjacent maxima or minima which are at wavelengths $\lambda_{1}$ and $\lambda_{2}$, the integer $m$ is equal to one. Then, (14) is modified, with the film thickness $d$ becoming the Swanepoel thickness $d_{S}$ [13], and therefore

$$
d_{S}=\frac{1}{2} \frac{\lambda_{1} \lambda_{2}}{\lambda_{1} n_{2}-\lambda_{2} n_{1}},
$$

where $n_{1}$ and $n_{2}$ are the refractive indices at wavelengths $\lambda_{1}$ and $\lambda_{2}$, respectively.

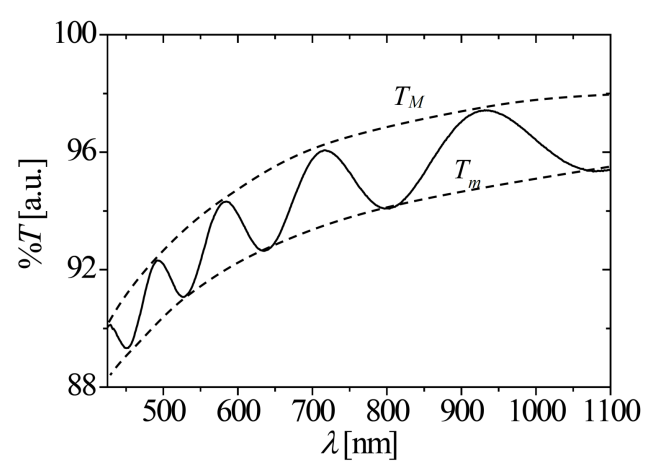

Fig. 2. Transmittance, $\% T$, vs wavelength, $\lambda$, of a thin polymer film showing an interference fringe pattern.

Once the transmittance contains a wave-like interference pattern, two adjacent maxima or minima can be used to calculate the film thickness, however which equations to use depends on the absorption region that the transmittance falls into. For instance, to calculate $n$ for the transparent absorption region, one uses (8), while (11) is valid for the weak absorption region. Once a pair of refractive indices is obtained, the Swanepoel thickness $d_{S}$ can be calculated by using (15).

\section{Experimental}

To calculate the thicknesses of polymer films by using the Swanepoel model, the films made from poly(methyl methacrylate), or PMMA, a polymer commonly used as a dielectric layer in organic-based electronic devices [25, 26], were fabricated under different conditions. PMMA from Sigma Aldrich (Singapore), was dissolved in butyl acetate with four different concentrations (i.e., $6 \%, 7 \%, 8 \%$ and $9 \%$ ) of weight/volume. Each solution was spincoated, by using an MTI VTC-50 spin-coater, onto a cleaned glass substrate of sizes $2 \times 2 \mathrm{~cm}^{2}$. The spin-coating process comprised two steps: The first step was fixed at $500 \mathrm{rpm}$ for $10 \mathrm{~s}$ while the second step was 20 s-long but at different 5 speeds, starting from $1000 \mathrm{rpm}$ to $3000 \mathrm{rpm}$ with an increment of $500 \mathrm{rpm}$. This gave five samples in each concentration, and 20 samples in all concentrations. After spin-coating, each sample was heated at $80^{\circ} \mathrm{C}$ for $30 \mathrm{~min}$ to dry out the residue solution before being collected in a dry, light-protected container.

Two sets of samples were fabricated. For the first set, one day after production, the films were characterised for their transmittance spectrum over the UV-visible region by using a Perkin Elmer Lambda 365 UV-Vis spectrometer from $420 \mathrm{~nm}$ to $1100 \mathrm{~nm}$ with a step of $1 \mathrm{~nm}$. The transmittance spectrum of this set was then used to calculate its Swanepoel thicknesses. For the second set, one day after production, the films were measured for their actual thicknesses by using a Bruker Dektak XT profilometer. 


\section{Results and discussion}

4.1. Effects of spin-coating speed and solution concentration on film thicknesses

Figure 3a shows the relation between the 2nd-step spin-coating speed $\omega$ and the average actual film thickness $d_{A \text {, avg }}$ obtained from the profilometer at each solution concentration. In turn, Fig. 3b shows the relation between the solution concentration and the average actual film thickness $d_{A \text {, avg }}$ at each spincoating speed. The value of $d_{A \text {,avg }}$ was averaged from multiple values of $d_{A}$ over the profile length of $1 \mathrm{~mm}$.

In Fig. 3a, it was observed that once the speed increased, the film thickness decreased. In Fig. 3b, the trend was reversed - the increase in concentration caused the increase in film thickness. These behaviors can be explained based on the film-forming mechanism in the spin-coating method. Two governing forces of the liquid during the spin coating are the viscous force and the centripetal force. In general, the film will form once both forces are balanced [27, 28]. However, once the spin-coating speed is increased, the centripetal force becomes stronger. Less liquid could then adhere to the substrate, resulting in a thinner film at higher speed, as shown in Fig. 3a [29]. On the contrary, when the film concentration increases, the solution is more viscous. The viscous force would then become stronger and hence more liquid could adhere to form the film, causing a thicker film, as shown in Fig. 3b.

\subsection{Film transmittance spectrum and thickness profile}

Figure $4 \mathrm{a}-\mathrm{d}$ shows the transmittance spectrum from $420 \mathrm{~nm}$ to $1100 \mathrm{~nm}$ of the films with the concentrations of $6 \%, 7 \%, 8 \%$ and $9 \%$, respectively, at different spin-coating speeds. The transmittance spectrum of all films exhibited a high transmittance greater than $90 \%$ over the visible region. These results corresponded to the highly transparent nature of the films that is a typical characteristic of thin PMMA films [9, 30], and they confirmed that the films are suitable for the Swanepoel thickness calculation [31]. Each spectrum contained a wave-like characteristic showing the existence of the interference between the transmitted rays of different path lengths as a result of multiple reflections in the film. The spectrum also indicated that the film's thicknesses were appropriate to be calculated by using the Swanepoel method [13].

Figure 5a-d shows the $1 \mathrm{~mm}$-long profiles of the film thickness $d_{A}$ with the concentration of $6 \%, 7 \%$, $8 \%$ and $9 \%$, respectively, at different spin-coating speeds. Each profile was characterised, starting from the center of the film which was, as expected, the location where the film's transmittance was obtained in the UV-Vis spectrometer. We used the
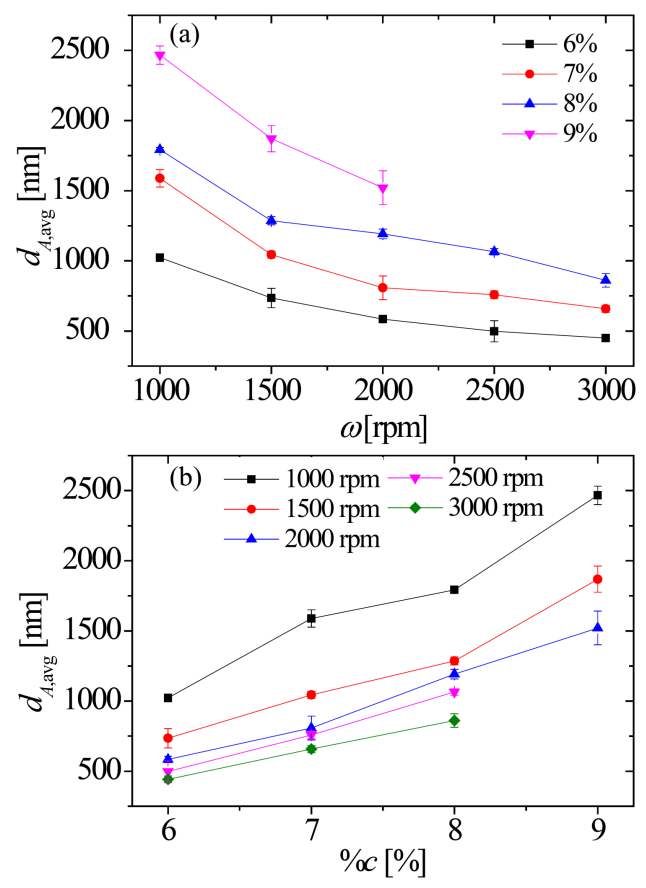

Fig. 3. Relations between (a) the 2nd-step spincoating speed $\omega$ and the average actual film thickness $d_{A \text {, avg }}$ at each solution concentration, and (b) the solution concentration \%c and the average actual film thickness $d_{A \text {,avg }}$ at each 2 nd-step spincoating speed.

profile length of $1 \mathrm{~mm}$ because it was the approximated diameter of the incident light beam used in the spectrometer.

\subsection{Comparison between Swanepoel thickness and actual thickness}

The wave-like transmittance spectrum was subsequently used to calculate the Swanepoel thickness. For a film with a specific concentration and a specific spin-coating speed, the calculation process started where the region selected for the Swanepoel model was the weak absorption one, with the model as shown by (10)-(15). This weak absorption model was considered as appropriate in this case because the transmittances of most films were rather high but still did not reach $100 \%$.

To apply the model, firstly, the transmittance spectrum was swept from high to low wavelengths to find the peaks and troughs of the wave-like spectrum. The wavelength for the first peak, or the first trough, depending on which one was first found, was selected as $\lambda_{1}$. Then the adjacent peak or trough, if $\lambda_{1}$ was a peak or trough, respectively, was selected as $\lambda_{2}$. As the spectrum was swept from high to low wavelengths, $\lambda_{2}$ was lower than $\lambda_{1}$. For both $\lambda_{1}$ and $\lambda_{2}$, their corresponding upper and lower envelope transmittances, $T_{M}$ and $T_{m}$, respectively, were recorded. Both values, together with the refractive index of the substrate $s$, were used to calculate the film refractive index $n$ following (11) and (12). Here, the refractive index $s$ of the glass substrate 

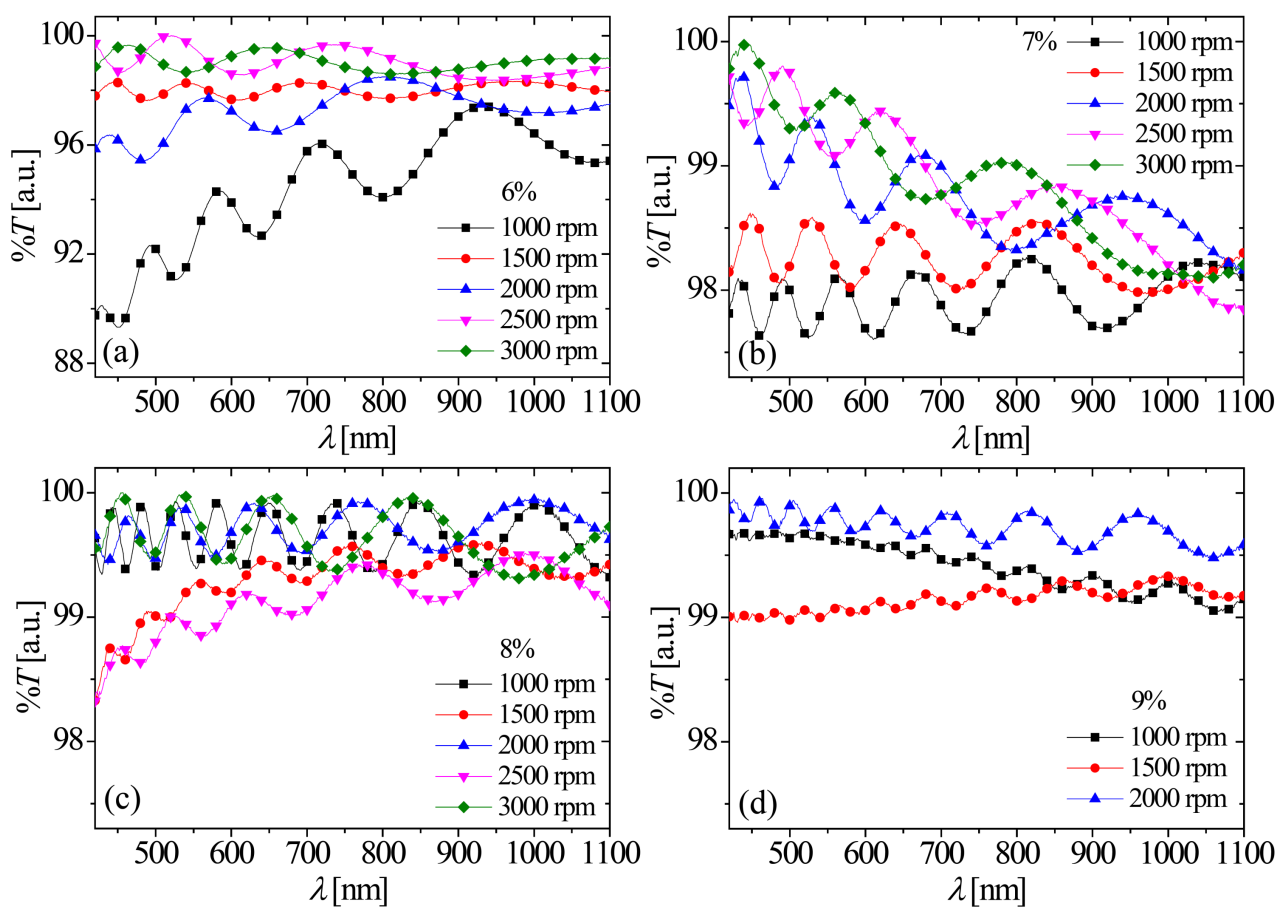

Fig. 4. Transmittance spectra of the films at the solution concentration of (a) $6 \%$, (b) $7 \%$, (c) $8 \%$, and (d), $9 \%$ at different 2 nd-step spin-coating speeds.
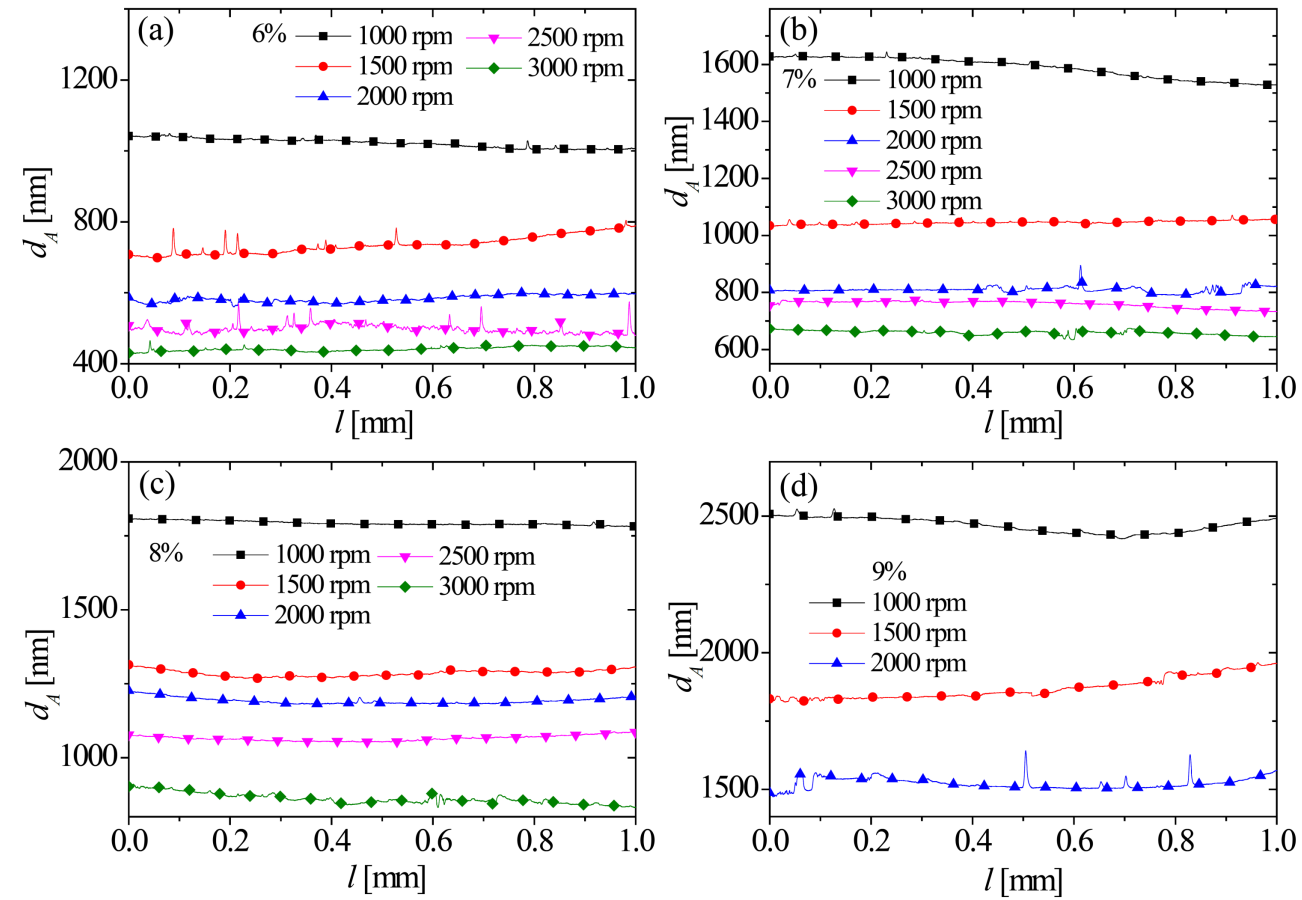

Fig. 5. Thickness profile $d_{A}$ along the length $l$ of the films at the solution concentration of (a) $6 \%$, (b) $7 \%$, (c) $8 \%$, and (d) $9 \%$ at different 2 nd-step spin-coating speeds.

was equal to 1.5 , as declared by the manufacturer. The two wavelengths, $\lambda_{1}$ and $\lambda_{2}$, consequently resulted in two refractive indices, $n_{1}$ and $n_{2}$, respectively. Finally, the Swanepoel film thickness $d_{S}$ for the $\lambda_{1}$ and $\lambda_{2}$ pair was calculated by using (15).
Most of the transmittance spectra in Fig. 4 contained more than one pair of peaks or troughs. Since the multiple pairs of $\lambda_{1}$ and $\lambda_{2}$ appeared, hence multiple Swanepoel thicknesses $d_{S}$ for a film could be calculated. These were then averaged and became 
TABLE I

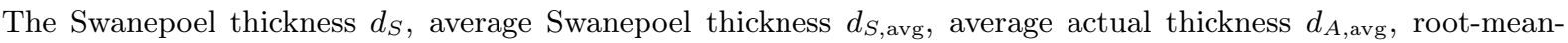
square roughness $R_{q}$, and percentage error \%err, at different pairs of $\lambda_{1}$ and $\lambda_{2}$ of films at $6 \%$ concentration at different spin-coating speeds.

\begin{tabular}{|c|c|c|c|c|c|c|c|}
\hline Speed [rpm] & $\lambda_{1}[\mathrm{~nm}]$ & $\lambda_{2}[\mathrm{~nm}]$ & $d_{S}[\mathrm{~nm}]$ & $d_{S, \text { avg }}[\mathrm{nm}]$ & $d_{A, \text { avg }}[\mathrm{nm}]$ & $R_{q}[\mathrm{~nm}]$ & \%err [\%] \\
\hline \multirow{4}{*}{1000} & 931 & 716 & 969.15 & \multirow{4}{*}{$1038.26 \pm 69.11$} & \multirow{4}{*}{$1022.13 \pm 26.60$} & \multirow{4}{*}{12.70} & \multirow{4}{*}{1.58} \\
\hline & 797 & 634 & 1100.32 & & & & \\
\hline & 716 & 585 & 1027.48 & & & & \\
\hline & 634 & 527 & 1056.08 & & & & \\
\hline \multirow{4}{*}{1500} & 966 & 695 & 760.61 & \multirow{4}{*}{$827.08 \pm 105.81$} & \multirow{4}{*}{$734.84 \pm 69.13$} & \multirow{4}{*}{24.25} & \multirow{4}{*}{12.55} \\
\hline & 810 & 609 & 803.27 & & & & \\
\hline & 695 & 541 & 932.89 & & & & \\
\hline & 609 & 489 & 811.54 & & & & \\
\hline \multirow{3}{*}{2000} & 1001 & 656 & 590.45 & \multirow{3}{*}{$593.18 \pm 2.73$} & \multirow{3}{*}{$583.97 \pm 23.50$} & \multirow{3}{*}{9.77} & \multirow{3}{*}{1.58} \\
\hline & 806 & 564 & 595.85 & & & & \\
\hline & 656 & 483 & 593.22 & & & & \\
\hline \multirow{3}{*}{2500} & 950 & 617 & 609.33 & \multirow{3}{*}{$566.13 \pm 43.20$} & \multirow{3}{*}{$498.30 \pm 75.95$} & \multirow{3}{*}{12.64} & \multirow{3}{*}{13.61} \\
\hline & 739 & 521 & 565.27 & & & & \\
\hline & 617 & 455 & 523.79 & & & & \\
\hline \multirow{2}{*}{3000} & 814 & 542 & 609.77 & \multirow{2}{*}{$576.95 \pm 32.84$} & \multirow{2}{*}{$441.94 \pm 23.06$} & \multirow{2}{*}{6.05} & \multirow{2}{*}{30.56} \\
\hline & 642 & 463 & 544.13 & & & & \\
\hline
\end{tabular}
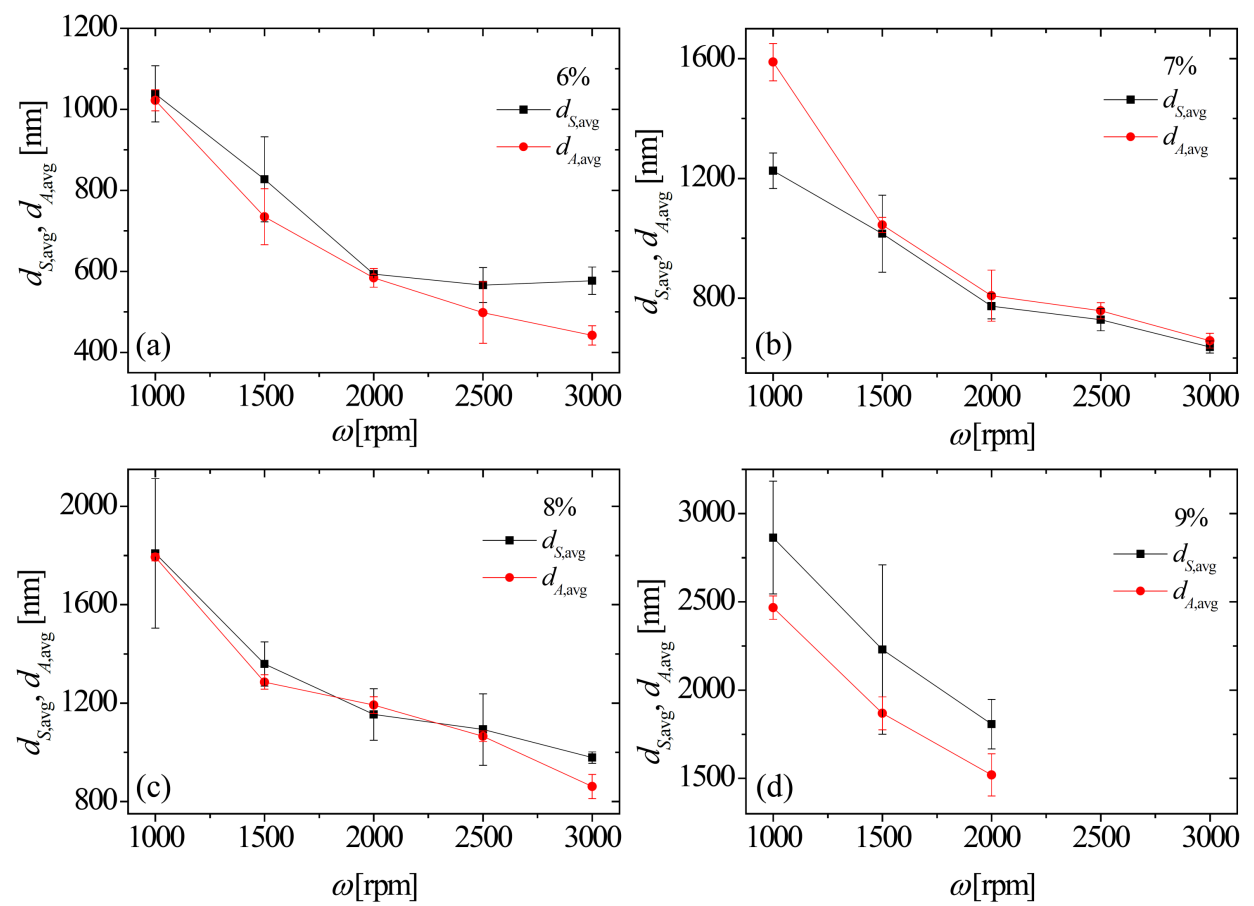

Fig. 6. Comparison of average Swanepoel thickness $d_{S, \text { avg }}$ and average actual thickness $d_{A \text {,avg }}$ of the films at different 2nd-step spin-coating speeds $\omega$ at the solution concentration of (a) $6 \%$, (b) $7 \%$, (c) $8 \%$, and (d) $9 \%$.

the average Swanepoel thickness $d_{S \text {,avg }}$ with its corresponding uncertainty. The given $d_{S \text {,avg values }}$ were then compared with $d_{A \text {,avg }}$ of the same condition, as shown in Fig. 6a-d for the films at concentrations of $6 \%, 7 \%, 8 \%$ and $9 \%$, respectively. The values of $\lambda_{1}, \lambda_{2}, d_{S}, d_{S, \text { avg }}$ and $d_{A \text {,avg, ob- }}$ tained from the $6 \%$-concentration films, are shown in Table I.
One could observe that the trends of $d_{S \text {,avg fol- }}$ lowed those of $d_{A \text {,avg }}$ for all films. Moreover, both values of most films matched each other within the ranges of their corresponding errors. This observation preliminarily showed how accurate $d_{S \text {, avg }}$ was

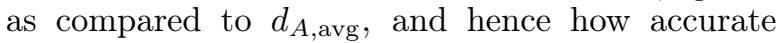
the Swanepoel method can be when calculating the thickness of polymer films. 


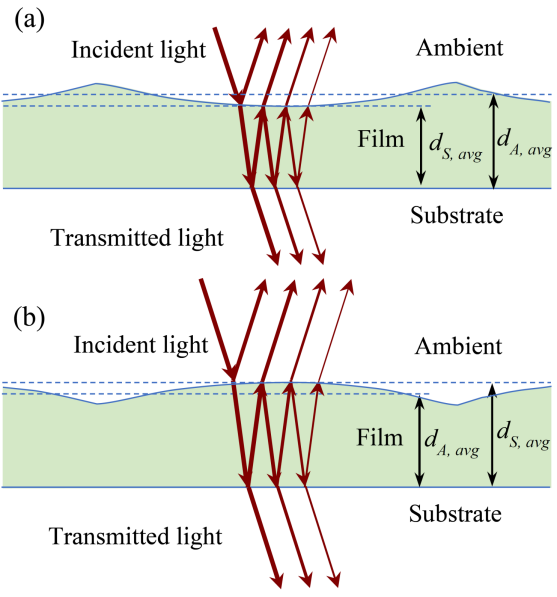

Fig. 7. Schematic diagrams of the multiple reflections of the film whose top surface is (a) pit- and (b) granule-dominated.

The relative difference between $d_{S \text {,avg }}$ and $d_{A \text {,avg }}$ can be explained regarding the region of the film where the interference of transmitted rays occurs. The interference pattern on a transmission spectrum is a result of multireflections inside a film with ideally flat top and bottom surfaces, as shown in Fig. 1b. However, the top surface of a spin-coated polymer film is not flat and typically consists of features including pits and granules [32, 33], giving rise to surface roughness [32]. For the pitted surface as shown in Fig. 7a, the flat region, where the interference occurs, is thinner than the average actual thickness $d_{A \text {,avg }}$ of the film. Thus, if the top surface of the film is pit-dominated, $d_{S \text {, avg }}$ is lower

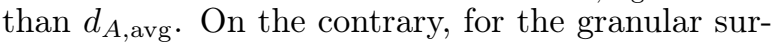
face as shown in Fig. 7b, such a flat region is thicker than $d_{A \text {,avg. }}$. Therefore, if the top surface is granuledominated, $d_{S \text {, avg }}$ is higher than $d_{A \text {,avg }}$.

To further analyse the difference between $d_{S \text {,avg }}$ and $d_{A, \text { avg }}$, the percentage error (\%err) between both values of each film was calculated using

$$
\% \text { err }=\frac{\left|d_{S, \text { avg }}-d_{A, \text { avg }}\right|}{d_{A, \text { avg }}} \times 100 \% .
$$

The correlations of the percentage error with the film's properties, namely the film roughness and thickness, were investigated. The former is a wellknown cause of inaccuracy of the Swanepoel thickness as it affects the absorption coefficient $\alpha$ and hence the refractive index $n$ of the film [20]. To analyse its effect on the accuracy of the Swanepoel thickness, the root-mean-square surface roughness $R_{q}$ of each film was calculated as follows [34]:

$$
R_{q}=\sqrt{\frac{\sum_{i=1}^{n_{\mathrm{tot}}}\left(d_{A, i}-d_{A, \mathrm{avg}}\right)^{2}}{n_{\mathrm{tot}}}} .
$$

Here, $d_{A, i}$ is the actual thickness measured at the $i$ point along the $1 \mathrm{~mm}$-length profile, $n_{\text {tot }}$ is the total number of measuring points. The values of $R_{q}$ and $\%$ err of the $6 \%$-concentration films are shown in Table I.

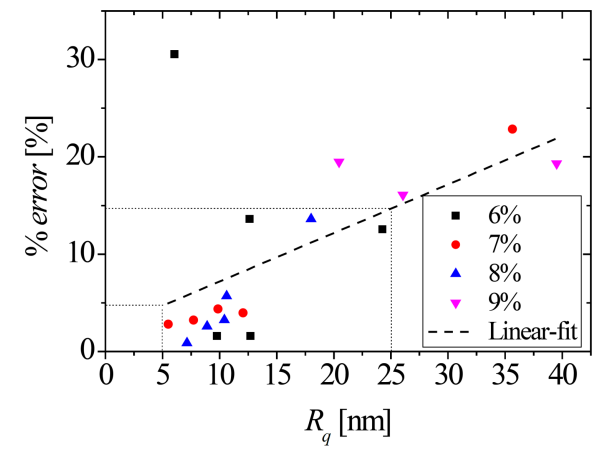

Fig. 8. Relation between the root-mean-square surface roughness $R_{q}$ and its corresponding percentage error \%err.

Figure 8 shows the dependence between $R_{q}$ and \%err of all the films considered in this paper. It could be observed that this $R_{q}$ parameter affected the error perceptibly. When $R_{q}$ was low, i.e., lower than $15.0 \mathrm{~nm}$, \%err values were as low as $5.0 \%$. For a film with higher $R_{q}$, \%err tended to increase. When the scattered data was linearly fitted, one observed that a fivefold increase in $R_{q}$ from $5.0 \mathrm{~nm}$ to $25.0 \mathrm{~nm}$ corresponded to a threefold increase in \%err from $4.9 \%$ to $14.7 \%$. For the range of our investigation, the films with the highest $R_{q}$ of around $40.0 \mathrm{~nm}$ had \%err of $20.0 \%$.

Mathematically, the effects of $R_{q}$ on the deviation in the Swanepoel thickness can be explained as follows [20]: When the average actual film thickness $d_{A \text {,avg }}$ is not uniform and can be represented as $d_{A \text {,avg }} \pm R_{q}$, then (1) is affected, resulting in the decrease in $T_{M}$ and increase in $T_{m}$, and so the interference pattern dramatically shrinks as compared to that obtained from a uniform film. This leads to the decrease in $N$ and consequently $n$, given by (12) and (11), respectively. Finally, $d_{S}$ calculated by (15) would be distorted from $d_{A \text {,avg. The }}$ greater $R_{q}$, the greater distorted $d_{S}$ from $d_{A \text {,avg. }}$.

To analyse whether the property (film thickness) had a crucial effect on the accuracy of the modelled thickness, we have shown $d_{A \text {,avg }}$ of each film versus its \%err in Fig. 9. Noticeably, the values of \%err were scattered over the whole range of $d_{A \text {,avg }}$ with a slightly increasing trend. When one made a linear fit to the data, it was found that a fivefold increase in $d_{A \text {,avg }}$ from $500 \mathrm{~nm}$ to $2500 \mathrm{~nm}$ corresponded to a 1.6 times increase of \%err from $8.3 \%$ to $13.4 \%$. The increasing trend of \%err with

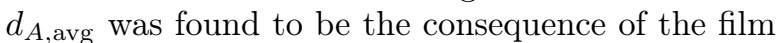
roughness. As demonstrated in the inset of Fig. 9 showing $d_{A \text {,avg }}$ vs. $R_{q}$, the roughness increased with $d_{A, \text { avg }}$. This indicated that the thickest films corresponded to high roughness, hence high error. However, if a thick film was smooth, its error was low. Based on this, it can be observed that a rather thick film with the thickness of $1800 \mathrm{~nm}$ had \%err as small as $0.86 \%$ because its roughness was only $7.1 \mathrm{~nm}$. This indicated that increasing the film thickness 


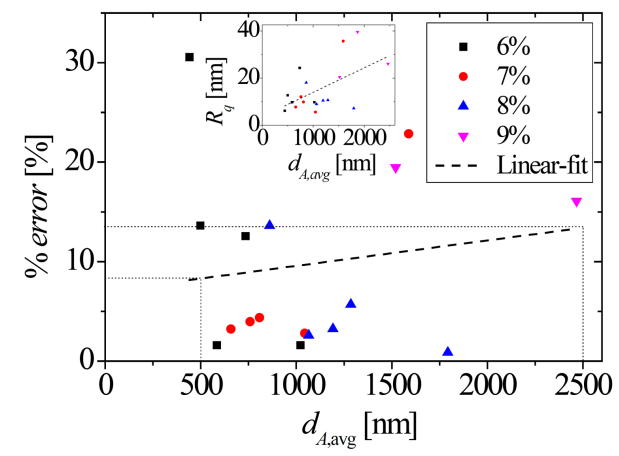

Fig. 9. Relation between the average actual film thickness $d_{A \text {,avg }}$ and its corresponding percentage error \%err. The inset shows the relation between the average actual film thickness $d_{A \text {,avg }}$ and its corresponding root-mean-square surface roughness, $R_{q}$.

did not significantly increase the inaccuracy in the modelled thickness of these polymer films, providing that the film's roughness was low.

Comparatively, the fivefold increases in film roughness and thickness corresponded to the threefold and 1.6 times increases in \%err, respectively. This result, along with the fact that the thickness of the film was probably governed by the surface roughness, implies that the accuracy of the Swanepoel model for polymer films relies much more on the roughness of the film than on its thickness. Thus, the rougher the film, the more distorted the Swanepoel thickness from the actual one. In this paper, most of the films with high surface roughness were those with the highest concentration at $9 \%$. This was because high-concentration solutions generally have high viscosity, leading to a film with greater surface roughness $[35,36]$. The films at this concentration were also the ones with high \%err, as observed in Fig. 9. This indicated that the Swanepoel model may not be suitable to calculate the thickness of spin-coated films from a high solution concentration as it may be highly inaccurate.

\section{Conclusions}

The Swanepoel method could be used to estimate the thickness of thin polymer films whose transmittances exhibited a wave-like pattern with error of less than $5.0 \%$, providing that the film surface roughness was lower than $15.0 \mathrm{~nm}$. The value increased with the roughness - the rougher the film, the greater the error. Increasing the surface roughness fivefold increases the error threefold. This error was due to the pitted and granular characteristics of the polymer films, making the modelled thickness lower and higher, respectively, than the average actual thickness. Regarding the film thickness, the parameter slightly correlated with the error. However, as the thickness corresponded with the roughness, and also films with the thickness greater than $1 \mu \mathrm{m}$ with the error of as low as $0.86 \%$ could be found, this indicated that the film thickness had insignificant effect on the method's accuracy. Thus, the use of the Swanepoel method to calculate the polymer film thickness was appropriate to a $\mu \mathrm{m}$-thick film with a good level of roughness.

\section{Acknowledgments}

K.W. would like to thank the College of Advanced Manufacturing Innovation for the funding scheme.

\section{References}

[1] O.G. Gnonhoue, A. Velazquez-Salazar, E. David, I. Preda, Polymers 13, 766, (2021).

[2] N. Andam, S. Refki, H. Ishitobi, Y. Inouye, Z. Sekkat, Photonics 8, (2021).

[3] S. Hernández-López, E. Vigueras-Santiago, M. Mayorga-Rojas, D. Reyes-Contreras, J. Phys. Conf. Ser. 167, 012059 (2009).

[4] H.-D. Liu, Y. Zhao, G. Ramanath, S.P. Murarka, G.-C. Wang, Thin Solid Films 384, 151 (2001).

[5] Ran Yi, ZhiDong Lou, YuFeng Hu, ShaoBo Cui, Feng Teng, YanBing Hou, XiaoJun Liu, Sci. China Technol. Sci. 57, 1142 (2014).

[6] M.R. Cavallari, L.M. Pastrana, C.D. Flecha Sosa, A.M. Rodriguez Marquina, J.E. Eirez Izquierdo, F. Josepetti Fonseca, C.A. de Amorim, L.G. Paterno, I. Kymissis, Materials 14, 3 (2021).

[7] L. Qi, L. Petersson, T. Liu, J. Int. Counc. Electr. Eng. 4, 1 (2014).

[8] A.N. Al-Omari, K.L. Lear, IEEE Trans. Dielectr. Electr. Insul. 12, 1151 (2005).

[9] N. Chapman, M. Chapman, W.B. Euler, Coatings 11, 198 (2021).

[10] Haihua Zhou, Weicai Liu, Rui Chang, Zhandong Huang, Xuzheng Sha, Guozhu Chen, E. Reichmanis, Yanlin Song, ACS Appl. Mater. Interfaces 12, 27786 (2020).

[11] O.D. Volpian, I.M. Drozd, A.I. Kuzmichev, Y.A. Obod, J. Phys. Conf. Ser. 872, 012028 (2017).

[12] H. Huang, L. Jiang, Y. Yao, Z. Zhang, Z. Wang, R. Qi, Coatings 11, 599 (2021).

[13] R. Swanepoel, J. Phys. E Sci. Instrum. 16, 1214 (1983).

[14] H. Hekmatara, M. Seifi, S.M. Rozati, Acta Phys. Pol. A 125, 77 (2014).

[15] M.M. Abdel-Aziz, I.S. Yahia, L.A. Wahab, M. Fadel, M.A. Afifi, Appl. Surf. Sci. 252 (23), 8163 (2006). 
[16] A. Kafizas, C. Crick, I.P. Parkin, J. Photochem. Photobiol. A 216, 156 (2010).

[17] D.A. Minkov, J. Phys. D Appl. Phys. 22, 1157 (1989).

[18] E.R. Shaaban, I.S. Yahia, E.G. El-Metwally, Acta Phys. Pol. A 121, 628 (2012).

[19] E.R. Shaaban, M.Y. Hassaan, M.G. Moustafa, A. Qasem, G.A.M. Ali, E.S. Yousef, Acta Phys. Pol. A 136, 498 (2019).

[20] R. Swanepoel, J. Phys. E Sci. Instrum. 17896 (1984).

[21] M. Ezzeldien, Z.A. Alrowaili, M.F. Hasaneen, Opt. Mater. 113, 110814 (2021).

[22] P. Prepelita, F. Garoi, V. Craciun, Beilstein J. Nanotechnol. 12, 354 (2021).

[23] O. Akpinar, A.K. Bilgili, U.C. Baskose, M.K. Ozturk, S. Ozcelik, E. Ozbay, J. Mater. Sci. Mater. Electron. 31, 9969 (2020).

[24] T.G. Mayerhofer, S. Pahlow, J. Popp, Chemphyschem 21, 2029 (2020).

[25] K. Budzalek, Hangjun Ding, L. Janasz, A. Wypych-Puszkarz, O. Cetinkaya, J. Pietrasik, M. Kozanecki, J. Ulanski, K. Matyjaszewski, J. Mater. Chem. C 9, 1269 (2021).
[26] T.-S. Huang, Y.-K. Su, P.-C. Wang, Jpn. J. Appl. Phys. 47, 3185 (2008).

[27] A.G. Emslie, F.T. Bonner, L.G. Peck, J. Appl. Phys. 29, 858 (1958).

[28] D. Meyerhofer, J. Appl. Phys. 49, 3993 (1978).

[29] N. Sahu, B. Parija, S. Panigrahi, Indian J. Phys. 83, 493 (2009).

[30] S. Fidan, Acta Phys. Pol. A 125, 494 (2014).

[31] Youliang Jin, Baoan Song, Zhitai Jia, Yinan Zhang, Changgui Lin, Xunsi Wang, Shixun Dai, Opt. Express 25, 440 (2017).

[32] Y. Li, Y. Yang, F. Yu, L. Dong, J. Polym. Sci. B 44, 9 (2006).

[33] N.G. Semaltianos, Microelectron. J. 38, 754 (2007).

[34] D. Pinto, A.M. Amaro, L. Bernardo, Appl. Sci. 10, 733 (2020).

[35] S.A. Kamaruddin, K.-Y. Chan, H.-K. Yow, M.Z. Sahdan, H. Saim, D. Knipp, Appl. Phys. A 104, 263 (2010).

[36] B.Ö. Uysal, Ö. Pekcan, Acta Phys. Pol. A 133, 1160 (2018). 